\title{
Social Saliency of the Cue Slows Attention Shifts
}

\author{
Vassiki Chauhan ${ }^{1}$, Matteo Visconti di Oleggio Castello' ${ }^{1}$, Alireza Soltani ${ }^{1}$ and \\ Maria Ida Gobbini ${ }^{1,2 *}$
}

${ }^{1}$ Department of Psychological and Brain Sciences, Dartmouth College, Hanover, NH, USA, ${ }^{2}$ Dipartimento di Medicina Specialistica, Diagnostica e Sperimentale, Medical School, University of Bologna, Bologna, Italy

Eye gaze is a powerful cue that indicates where another person's attention is directed in the environment. Seeing another person's eye gaze shift spontaneously and reflexively elicits a shift of one's own attention to the same region in space. Here, we investigated whether reallocation of attention in the direction of eye gaze is modulated by personal familiarity with faces. On the one hand, the eye gaze of a close friend should be more effective in redirecting our attention as compared to the eye gaze of a stranger. On the other hand, the social relevance of a familiar face might itself hold attention and, thereby, slow lateral shifts of attention. To distinguish between these possibilities, we measured the efficacy of the eye gaze of personally familiar and unfamiliar faces as directional attention cues using adapted versions of the Posner paradigm with saccadic and manual responses. We found that attention shifts were slower when elicited by

Edited by: Sebastian Loth,

Bielefeld University, Germany

Reviewed by:

Wendy E. Huddleston, University of Wisconsin-Milwaukee,

Agnieszka Wykowska Istituto Italiano di Tecnologia, Italy

*Correspondence:

Maria Ida Gobbin mariaida.gobbini@unibo.it; maria.i.gobbini@dartmouth.edu

Specialty section:

This article was submitted to Cognitive Science, a section of the journal

Frontiers in Psychology

Received: 14 November 2016 Accepted: 24 April 2017

Published: 15 May 2017

Citation:

Chauhan V, Visconti di Oleggio Castello M, Soltani A and Gobbini MI (2017) Social Saliency of the Cue Slows Attention Shifts.

Front. Psychol. 8:738. doi: 10.3389/fpsyg.2017.00738 a perceived change in the eye gaze of a familiar individual as compared to attention shifts elicited by unfamiliar faces at short latencies (100 ms). We also measured simple detection of change in direction of gaze in personally familiar and unfamiliar faces to test whether slower attention shifts were due to slower detection. Participants detected changes in eye gaze faster for familiar faces than for unfamiliar faces. Our results suggest that personally familiar faces briefly hold attention due to their social relevance, thereby slowing shifts of attention, even though the direction of eye movements are detected faster in familiar faces.

Keywords: personal familiarity, cue salience, social cues, gaze cueing, eye gaze, face processing, slowed disengagement of attention

\section{INTRODUCTION}

Social cues, such as direction of eye gaze and head angle, are effective in redirecting one's attention to salient aspects of the environment (Friesen and Kingstone, 1998; Driver et al., 1999; Langton and Bruce, 1999; Hoffman and Haxby, 2000; Pelphrey et al., 2003; Senju and Csibra, 2008; Senju and Johnson, 2009). Here, we investigated whether reallocation of spatial attention was faster in response to the shift in eye gaze of a familiar individual as compared to the shift in eye gaze of a stranger.

Several studies have demonstrated that perceived eye gaze spontaneously biases spatial attention in the direction of the gaze (Friesen and Kingstone, 1998; Driver et al., 1999; Langton and Bruce, 1999; Hoffman and Haxby, 2000; Pelphrey et al., 2005; Frischen et al., 2007; Senju and Csibra, 2008; Senju and Johnson, 2009). These biases in spatial attention have previously been shown to be susceptible to top-down influences (Teufel et al., 2010; Kawai, 2011; Wiese et al., 2012, 2014; 
Wykowska et al., 2014). The neural mechanisms underlying gaze perception have been studied extensively. fMRI studies have shown that specific regions, such as the posterior and anterior superior temporal sulcus, the intraparietal sulcus, the medial prefrontal cortex, are preferentially engaged by eye gaze and head angles, highlighting how dedicated populations of neurons are involved in processing these social cues (Hoffman and Haxby, 2000; Pageler et al., 2003; Pelphrey et al., 2003; Engell and Haxby, 2007; Carlin et al., 2012; Carlin and Calder, 2013; and for a review, Senju and Johnson, 2009). Interestingly, eye gaze and head position are processed even without awareness, underscoring that detection of these features, and the subsequent effect on spatial attention, can be preconscious and any facilitation or slowing by familiarity may be acting upon a very early stage of processing (Stein et al., 2011; Gobbini et al., 2013a).

Personally familiar faces are highly salient social stimuli (Gobbini and Haxby, 2007). Efficient face processing through multimodal experience with familiar others serves to facilitate interactions with those who are most important in our social lives. Familiarity affords more efficient face detection and recognition of identity (Bruce et al., 1994; Burton et al., 1999; Jenkins et al., 2011; Ramon et al., 2011; Gobbini et al., 2013b; Ramon, 2015; Visconti di Oleggio Castello and Gobbini, 2015; Ramon and Van Belle, 2016; Visconti di Oleggio Castello et al., 2016). Similarly, processing of social cues in faces, such as direction of attention, are markedly facilitated by familiarity. For example, in a study of perception of gaze direction and head angle, perception of eye gaze was detected around $100 \mathrm{~ms}$ faster in familiar as compared to the faces of strangers (Visconti di Oleggio Castello et al., 2014).

Here, we investigated whether personal familiarity with the faces whose change in eye gaze served as attentional cue has an influence on how we reallocate spatial attention in response to those eye gaze changes. We used a gaze cueing paradigm - an adaptation of the Posner cueing paradigm (Posner, 1980). In the Posner cueing paradigm, presentation of a directional cue (e.g., an arrow) precedes the onset of a target in either the cued or uncued direction. Participants are faster and more accurate in processing a target if it appears in the cued direction (Posner, 1980). In the gaze cueing paradigm, faces with directional eye gaze served as the directional cue for manipulating spatial attention. Perceived eye gaze has proved successful in modulating spatial attention in schematic faces (Friesen and Kingstone, 1998). One could hypothesize that facilitation of detection of eye gaze direction in personally familiar faces leads to more effective redirection of spatial attention as compared to detection of eye gaze shifts in the faces of strangers (Visconti di Oleggio Castello et al., 2014). As an alternative hypothesis, highly salient faces of familiar individuals could hold attention (Bindemann et al., 2005), thus making redirecting spatial attention slower, similar to a highly rewarding feature in visual search tasks (Hickey et al., 2010; Anderson et al., 2011; Hickey and Peelen, 2015; Munneke et al., 2015). Allocation of attentional resources is driven by relative salience of stimuli encountered in the environment, where high salience can be generated by the relative configuration of low-level features such as color, orientation and size in the scene (Itti and Koch, 2001; Soltani and Koch, 2010) or by an interaction between sensory features and reward (Markowitz et al., 2011; Theeuwes and Belopolsky, 2012; Khorsand et al., 2015). Similarly, valence of the face cue (e.g., faces with expression of emotion such as fear, happy, neutral, or sad or one's own face; Pourtois et al., 2013; Pérez-Duenas et al., 2014; Porciello et al., 2014) has been shown to modulate the magnitude of attentional capture. Thus, spatial reallocation of attention in response to gaze shifts in familiar faces could be facilitated by faster processing of the gaze cue or slowed by the social saliency of familiar faces.

We tested these two competing hypotheses on how face familiarity interacts with redirection of attention in response to shifts in eye gaze with two experiments. In the first experiment, we asked participants to make a saccade to the target appearing in the periphery either on the right or on the left of a centrally presented face. In the second experiment, we asked the participants to report the side on which the target appeared with a button press, without looking away from the centrally presented face. We used the manual response paradigm to test whether slowed initiation of saccades to a target is attributable to the difficulty of breaking fixation from salient centrally presented faces. Finally, we performed a control experiment to ensure that results from the first two experiments on reallocation of attention were not due to differences in detecting eye movements in personally familiar and unfamiliar faces (Visconti di Oleggio Castello et al., 2014). In this experiment, we measured participants' speed in detecting a change in the direction of eye gaze in personally familiar and unfamiliar faces.

\section{EXPERIMENT 1}

In Experiment 1, we investigated attention shifts elicited by the eye gaze of familiar and unfamiliar faces using a target-detection task based on the Posner cueing paradigm, with saccadic reaction time (SRT) as the dependent variable. Participants saw a directional gaze cue to the left or right in a familiar or unfamiliar face followed by a peripheral target that could appear on either side of the fixation cross. They were instructed to saccade toward the target as soon as it appeared on the screen. We manipulated the familiarity of the face cue, the congruence between the cue and target direction, and the delay between the cue and the target onset. Participants were instructed to be as fast as possible in their response, but not at the expense of accuracy.

\section{Materials and Methods Participants}

Fifteen students from the Dartmouth College community participated in Experiment 1 (seven male, Mean age: $26.4 \pm 3.22$ ). All participants were right handed with the exception of one. All participants provided written informed consent to participate in the experiment, and were paid. The Dartmouth Committee for the Protection of Human Subjects approved the experiment (Protocol 21200).

The number of participants was determined with a power analysis performed with the package 'pwr' in R (Champely, 2009) to ensure that the sample size was large enough to replicate the 
effect of gaze cueing reported previously (Kuhn and Kingstone, 2009). Other studies exploring the effects of gaze cueing have used similar sample sizes (Ricciardelli et al., 2002; Kuhn and Kingstone, 2009; Hungr and Hunt, 2012).

\section{Equipment}

All stimuli were presented on an FSI AM250 monitor, which has a refresh rate of $60 \mathrm{~Hz}$. The resolution of the display was $1920 \times 1080$ pixels. Eye movement data was collected with an Eyelink 1000 Plus Desktop Mount eye tracker system. Participants were seated $60 \mathrm{~cm}$ from the presentation screen throughout the course of the experiment, with their chin on a chin rest to minimize head movements.

\section{Stimuli}

Grayscale pictures of friends of the participants were used as familiar stimuli. All friends were students from the Dartmouth Community with whom participants had a good relationship for at least 1 year. Unfamiliar stimuli were pictures of age and gender matched controls that were taken at another college in an identical studio setting, using the same lighting and camera, to guarantee equivalent picture quality. For each participant, we used pictures of three friends and three strangers.

We used the following procedure to construct the face cue stimuli. We used a full face image with direct eye gaze of each identity as the base image, then superimposed an image of the pupil and iris from images of the same identity looking to the left or right. Minor smoothing with GIMP was performed to give the images a natural appearance. Thus, three images were constructed for each identity: eyes gazing forward, eyes gazing to the left, and eyes gazing to the right.

To avoid confounds due to low-level visual properties of stimuli, all the stimulus images were matched to the average luminance value of all the pictures and for contrast with the lumMatch function from the SHINE toolbox (Willenbockel et al., 2010).

\section{Experimental Paradigm}

Experiment 1 consisted of five blocks of 120 trials each, resulting in a total of 600 trials in the entire experiment. The experimental manipulations were Familiarity of the faces (Familiar or Unfamiliar), Validity of Cue (Congruent or Incongruent), and stimulus onset asynchrony (SOA) between the cue and target (100 or $200 \mathrm{~ms}$ ). These SOAs cover the initial stages of the effect of attention reallocation when it is building up and reaching its maximum. All three conditions were counterbalanced. Over the entire experiment, the picture of each individual identity was repeated 100 times. Version 3.0.12 of Psychtoolbox (Kleiner et al., 2007) on MATLAB 2014b was used for the purpose of stimulus presentation and response collection.

At the beginning of each block the eye-tracker was calibrated and validated with a five-point calibration. The trial started with a centrally presented black fixation cross. The fixation cross subtended $1 \times 1$ degree of visual angle around the center of the screen. Each trial started with a button press by the participant.
Following the button press, the fixation cross was replaced by the image of a face gazing forward. Each face image subtended $3 \times 4.3$ degrees of visual angle centered on the center of the screen. The forward gazing face remained onscreen for a jittered interval between 750 and $1000 \mathrm{~ms}$, in order to avoid a build-up of expectation for the gaze cue. This was followed by a gaze shift to the left or right, and after an SOA of either 100 or $200 \mathrm{~ms}$, a black target circle appeared in either the cued or uncued location. The gaze cue was valid for half the trials in each block and, thus, was uninformative. Others have shown (e.g., Friesen and Kingstone, 1998) that gaze cues do not have to be informative in order to induce reflexive shifts of attention. The target circle subtended half a degree of visual angle around a point that was 10 degrees away from the center of the screen. The gaze cue remained onscreen for the entire duration of the trial $(800 \mathrm{~ms})$.

Participants were instructed to maintain fixation on the centrally presented face for the period when the gaze was directed toward them and to continue looking at the face when the eye gaze changed direction. They were asked to move their eyes toward the black target circle as soon as it appeared on the left or right. They were instructed to respond as fast as possible but not at the expense of accuracy (Figure 1). They were told that direction of the eye gaze was not informative.

\section{Data Analysis}

In order to calculate SRT, we analyzed the subject's gaze position after the target was displayed on screen. We took the first time point at which the $x$ coordinate of the gaze position exceeded the borders of the centrally presented face to be the SRT. All the trials landed in a neighborhood of 1 degree of visual angle around the target. We marked the trials in which eye movements were made in the direction opposite to that of the target as incorrect trials. We did not include trials in which eye movements failed to land on the target in subsequent analyses ( $<2 \%$ of total trials).

We also marked trials in which eye gaze did not cross the image border in either direction as errors and included them in the calculation of accuracy. Finally, we discarded the trials that represented reaction times shorter than $80 \mathrm{~ms}$.

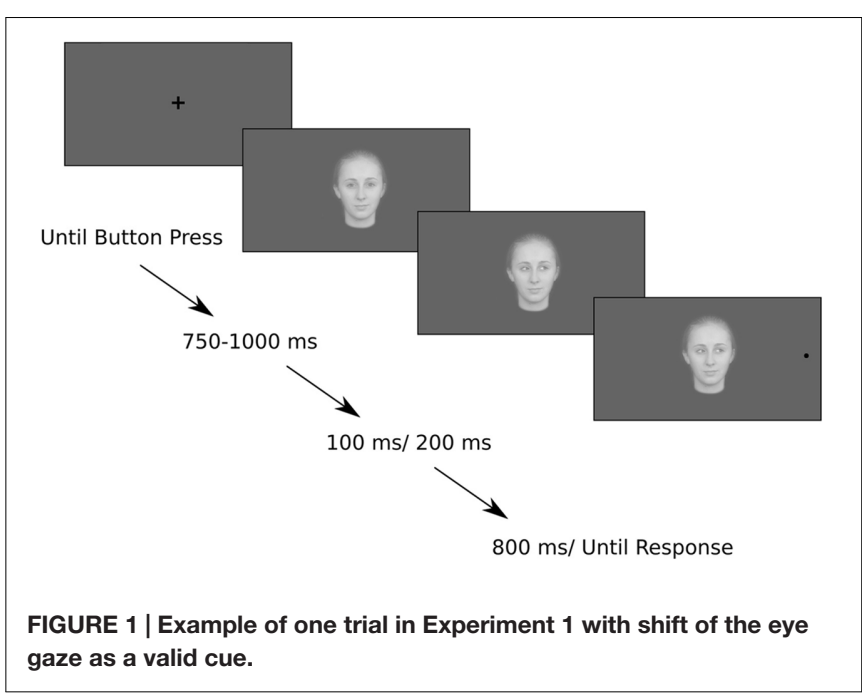


We used MATLAB for defining saccades and $\mathrm{R}$ ( $\mathrm{R}$ Core Team, 2013) for subsequent statistical analysis. We constructed a linear mixed model with log transformed SRT as the dependent variable, familiarity condition, validity and the SOA as fixed effects and participants as random effects. The reaction times were log transformed in order to fit the assumptions of linear mixed models. Package lme4 from CRAN was used for mixed models analysis (Bates et al., 2014). For both logit and linear mixed models, different models with random effects were created and compared with log likelihood ratio tests; the model that yielded the lowest Akaike's information criterion (AIC) was chosen. Once a final model was determined, statistical significance of the main and interaction effects was tested using a Type 3 Analysis of Deviance, as implemented in the package car (Fox and Weisberg, 2010). Note that an analysis of deviance tests the differences in deviance of a model using a chi-square test, and thus chi-square values are reported for both linear and logit models.

\section{Results}

We first analyzed the entire set of trials in order to assess if any of the experimental manipulations influenced the number of errors made throughout the course of the experiment. Using participants' response (correct or incorrect) as the dependent variable, and familiarity condition, cue validity, and SOA as predictors, we constructed a generalized linear mixed model with binomial error distribution and logit model as linking function (lme4 package). We found that the participants made significantly more errors on incongruent trials $\left[\chi^{2}(1)=121.35\right.$, $p<0.001]$ and, surprisingly, the longer SOA $\left[\chi^{2}(1)=93.92\right.$, $p<0.001]$ but the familiarity condition did not have a statistically significant effect on the number of errors made $\left[\chi^{2}(1)=0.31\right.$, $p=0.72]$ (Figure 2).

We then assessed whether the three experimental manipulations influenced the SRT when responding to the presence of the target. Our results revealed significant main effects of all three experimental manipulations. Firstly, we found a main effect of cue validity $\left[\chi^{2}(1)=99.98, p<0.001\right]$ showing shorter reaction times for valid as compared to invalid trials. Secondly, we found a main effect of SOA $\left[\chi^{2}(1)=83.44\right.$, $p<0.001]$ indicating slower responses for the shorter delay between the cue and target. Finally, we found a main effect of familiarity $\left[\chi^{2}(1)=9.75, p=0.002\right]$ with longer reaction times for familiar faces. Moreover, we found significant interactions of familiarity condition and SOA $\left[\chi^{2}(1)=18.84, p<0.001\right]$, and of cue validity and SOA $\left[\chi^{2}(1)=35.73, p<0.001\right]$. There was no significant interaction of cue validity and familiarity $\left[\chi^{2}(1)=1.06, p=0.30\right]$. The three-way interaction between validity, familiarity condition and SOA was significant $\left[\chi^{2}(1)=5.75, p=0.02\right]$. Saccades toward the target were slower on invalid trials than on valid trials, and this effect of validity was larger with the longer SOA between the cue and target. Moreover, the interaction between validity, familiarity condition, and SOA indicates that the effect of familiarity only holds if the delay between cue and target is $100 \mathrm{~ms}$. Thus, at the short SOA but not the long SOA participants were slower to saccade to both valid and invalid targets and the effect of cue validity was smaller if the cue was signaled by a familiar face as compared to a stranger's face (Figure 3). Effect sizes are provided in Tables 1-3.

\section{Interim Discussion}

We observed longer reaction times following familiar faces at the shorter delay between the cue and target onsets. These results suggest that participants are slower in looking away from familiar faces as compared to faces of strangers, thereby delaying the reaction time in response to the target both for valid and invalid gaze cues. We reasoned that the same results might not hold true if the task does not require the participant to explicitly look away from the centrally presented face. In order to test this hypothesis, we designed an experiment to test the effect familiarity of the cue on shifts of attention that do not involve saccades to the target.

\section{EXPERIMENT 2}

In this experiment, we investigated whether findings reported in Experiment 1 would hold if the response to the attended target did not involve explicit eye movements away from the centrally presented face cue, we tested the same participants in a paradigm that involved a manual response via a button press.

\section{Materials and Methods}

\section{Participants, Stimuli, and Equipment}

The stimuli and testing equipment were exactly the same as Experiment 1. Thirteen of the original 15 participants participated in this experiment (seven male, Mean age: $27.38 \pm 2.06)$. All participants provided written informed consent to participate in the experiments, and were paid. The Dartmouth Committee for the Protection of Human Subjects approved the experiment (Protocol 21200).

\section{Experimental Paradigm}

The sequence for presenting stimuli within a trial was exactly the same as in Experiment 1 (see Figure 1), except that we did not vary the SOA in this experiment-since results of Experiment 1 indicated that the effects of interest are present in the shorter delay (100 ms) between the cue and target. Participants performed three blocks of 100 trials each, resulting in a total of 300 trials. Over the course of the experiment, the picture of each individual identity was repeated 50 times. Eye movements were recorded to ensure that participants maintain central fixation (see Experiment 1 on details how eye movements were recorded). Participants responded with their dominant hand.

The task was similar to Experiment 1, except that participants were asked to respond manually by pressing the left or right arrow key to indicate the side where the black target circle appeared. The participants were instructed to maintain fixation on the centrally presented face and only respond when the target appeared in their peripheral vision. Trials in which eye movements were made in this period were discarded $(<1 \%)$. As in Experiment 1 , participants were instructed to respond as fast as possible but not at the expense of accuracy. 


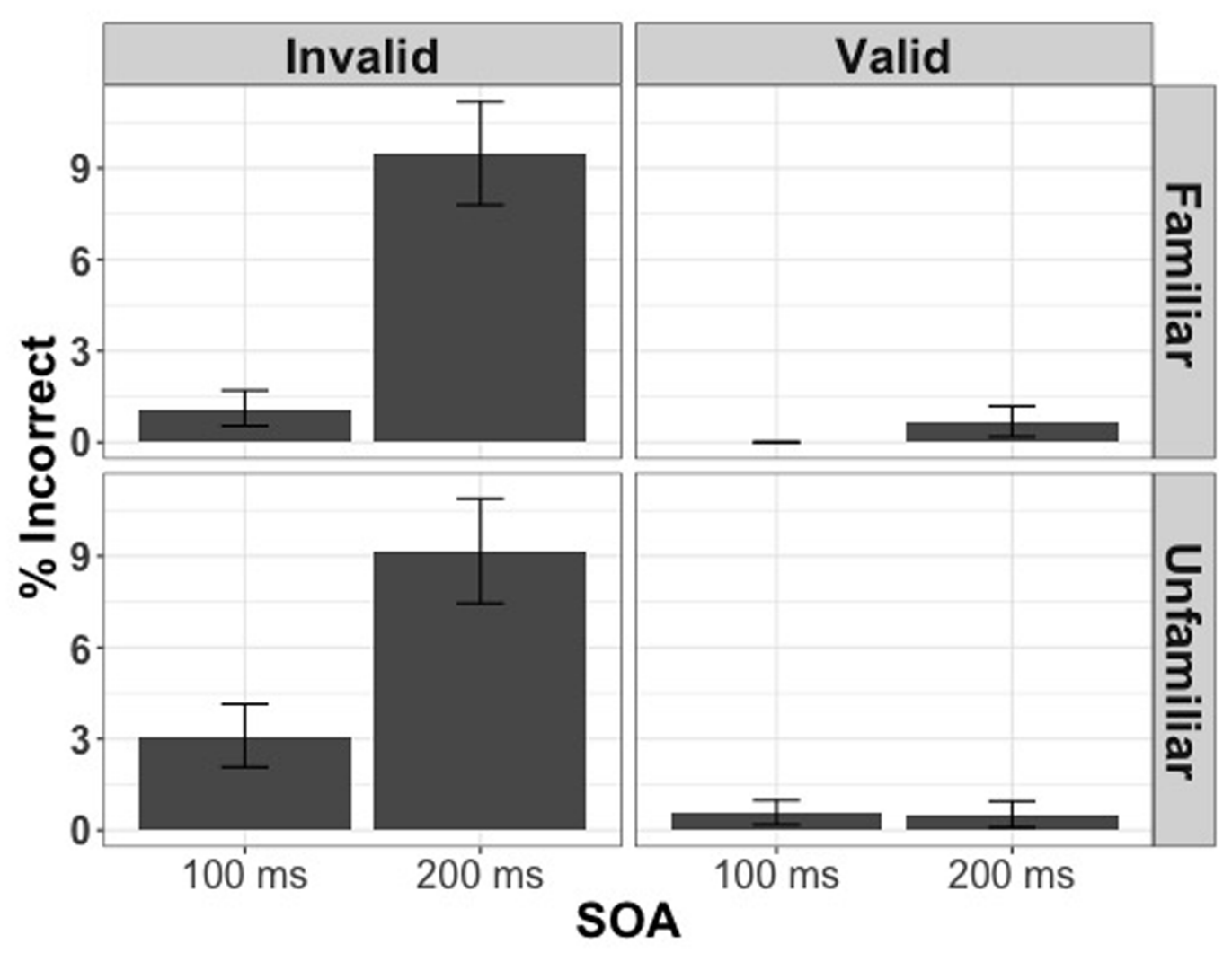

FIGURE 2 | Percentage of incorrect responses for 100 and 200 ms split by cue validity and familiarity condition in Experiment 1.

\section{Data Analysis}

We discarded trials in which reaction time was less than $100 \mathrm{~ms}$ as anticipatory responses. Moreover, we also removed trials in which eye movements were made as they reflected the failure to maintain fixation.

We constructed a linear mixed model with log transformed manual response time as the dependent variable, the familiarity condition and cue validity as the fixed effects and the participants as random effects. We created different models with random effects and compared them with log likelihood ratio tests and chose the model that yielded the lowest Akaike's information criterion (AIC) was chosen (see Data Analysis of Experiment 1). The values reported in the results were obtained from Type 3 Analysis of Deviance on each model, performed with the function ANOVA from package car (Fox and Weisberg, 2010).

\section{Results}

Analysis of the entire set of trials (including correct and incorrect responses) with accuracy as the dependent variable revealed that more incorrect responses were made for incongruent trials $\left[\chi^{2}(1)=21.32, p<0.001\right]$, but familiarity did not have an effect on the accuracy of responses $\left[\chi^{2}(1)=1.60, p=0.2\right]$ (Figure 4).

The linear mixed model constructed for reaction time as a function of familiarity condition and validity revealed significant main effects of cue validity $\left[\chi^{2}(1)=51.34, p<0.001\right]$ and familiarity $\left[\chi^{2}(1)=7.21, p=0.007\right]$ (Figure 5). The magnitude of the effect of familiarity in this Experiment $(5.5 \mathrm{~ms})$ was smaller than the effect of familiarity found at the $100 \mathrm{~ms}$ SOA of Experiment 1 (15 ms). Finally, as observed in Experiment 1, there was no significant interaction of cue validity and cue familiarity $\left[\chi^{2}(1)=0.98, p=0.32\right]$. Effect sizes for this experiment are reported in Tables 4,5 .

\section{Interim Discussion}

Results from Experiment 2 revealed an effect of familiarity on attention shifting on the timescale of $100 \mathrm{~ms}$, similar to what was found in Experiment 1 but with a smaller magnitude. In both experiments, reaction times to targets were slower for familiar faces. Altogether, we found effects of slowing of attentional disengagement by familiar face stimuli in both experiments, suggesting that familiar faces are highly salient stimuli that briefly hold attention, interfering with shifts of attention to other locations. In order to assess if these results arose from differences in processing the gaze cue itself in personally familiar and unfamiliar faces, we ran one more experiment. We assessed differences in processing eye gaze in familiar and unfamiliar faces in the absence of a task requiring a shift in spatial attention by asking participants to make a manual response to indicate the direction of eye gaze in familiar and unfamiliar faces. 


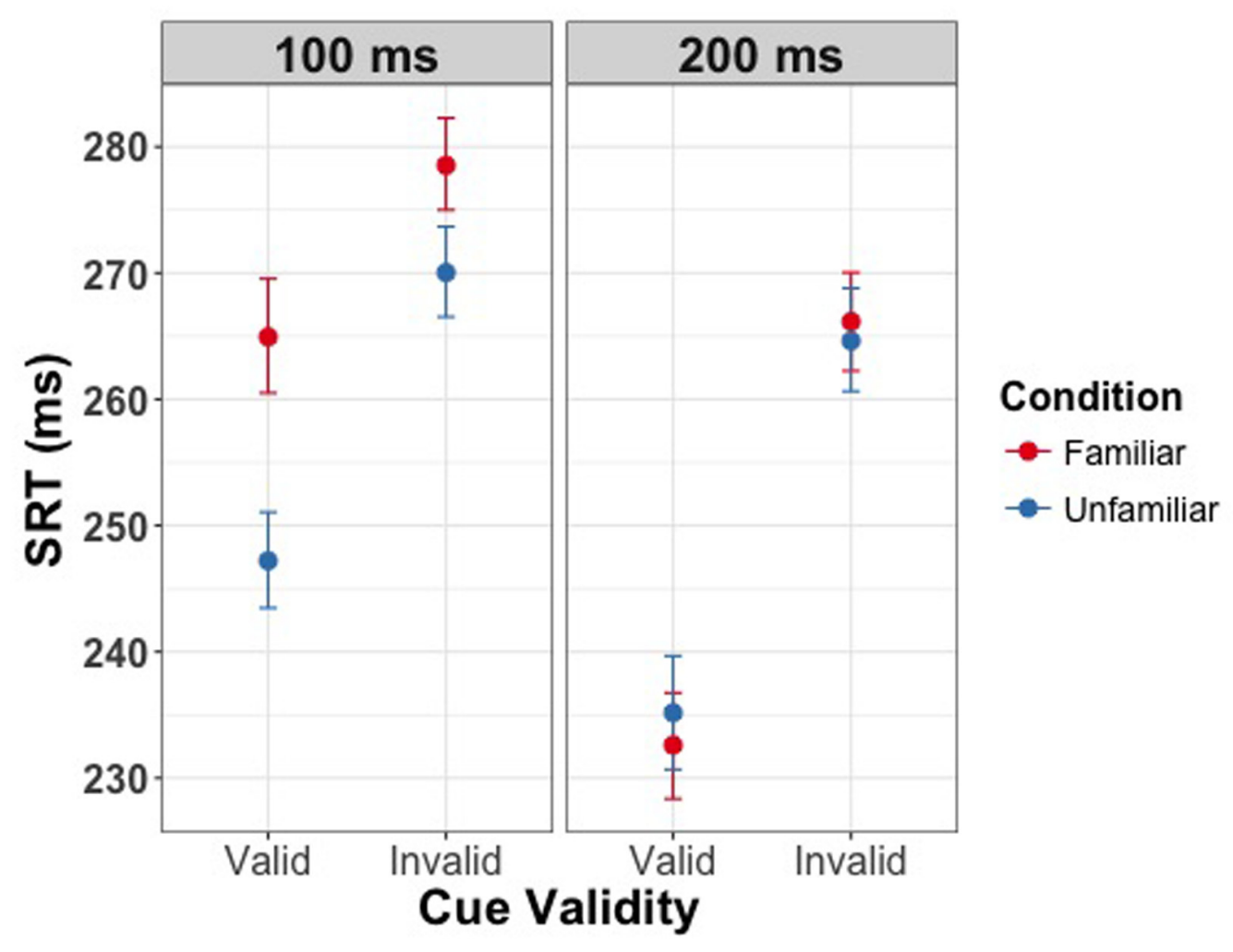

FIGURE 3 | Saccadic response time (SRT) as a function of validity of the eye gaze cue in Experiment 1. Left panel depicts the effect at the SOA of 100 ms, right panel depicts the effect at $200 \mathrm{~ms}$. Error bars represent bootstrapped $95 \%$ confidence intervals around the mean. Familiarity of the gaze cue results in longer latencies for saccadic response at an SOA of $100 \mathrm{~ms}$.

TABLE 1 | Main effect of familiarity in Experiment 1: Familiar RT - Unfamiliar RT.

\begin{tabular}{|c|c|c|c|c|c|}
\hline SOA & Validity condition & $\begin{array}{l}\text { Mean difference of } \\
\text { familiarity (ms) }\end{array}$ & $\begin{array}{l}\text { Pooled standard } \\
\text { deviation (ms) }\end{array}$ & Cohen's $d$ & $\begin{array}{c}\text { Confidence } \\
\text { interval }\end{array}$ \\
\hline $100 \mathrm{~ms}$ & Invalid & 8.42 & 57.2 & 0.15 & $0.06,0.26$ \\
\hline $100 \mathrm{~ms}$ & Valid & 17.6 & 63.1 & 0.28 & $0.18,0.40$ \\
\hline $200 \mathrm{~ms}$ & Invalid & 1.21 & 63.2 & 0.02 & $-0.06,0.12$ \\
\hline $200 \mathrm{~ms}$ & Valid & -2.06 & 72 & -0.03 & $-0.11,0.07$ \\
\hline
\end{tabular}

TABLE 2 | Main effect of cue validity in Experiment 1: Invalid RT - Valid RT.

\begin{tabular}{|c|c|c|c|c|c|}
\hline SOA & Familiarity condition & $\begin{array}{l}\text { Mean difference of } \\
\text { cue validity (ms) }\end{array}$ & $\begin{array}{l}\text { Pooled standard } \\
\text { deviation (ms) }\end{array}$ & Cohen's d & $\begin{array}{c}\text { Confidence } \\
\text { interval }\end{array}$ \\
\hline $100 \mathrm{~ms}$ & Familiar & 13.8 & 59.0 & 0.23 & $0.14,0.33$ \\
\hline $100 \mathrm{~ms}$ & Unfamiliar & 23.0 & 57.3 & 0.40 & $0.32,0.49$ \\
\hline $200 \mathrm{~ms}$ & Familiar & 32.2 & 60.0 & 0.54 & $0.46,0.64$ \\
\hline $200 \mathrm{~ms}$ & Unfamiliar & 29.0 & 63.1 & 0.46 & $0.38,0.56$ \\
\hline
\end{tabular}

\section{EXPERIMENT 3}

In order to ensure that the results reported in Experiments 1 and 2 did not come from differences in processing the eye gaze from familiar and unfamiliar faces, we asked participants to indicate the direction of eye gaze changes in familiar and unfamiliar faces with a manual response.

\section{Materials and Methods}

The stimuli and testing equipment were exactly the same as in Experiment 2.

Nine (four male, Mean age: $28.11 \pm 0.56$ ) of the original 13 participants from Experiment 2 participated in this experiment. Three of the previous participants had graduated and left the campus and one did not respond. All participants provided 
TABLE 3 | Main effect of SOA in Experiment 1: 100 ms SOA - 200 ms SOA.

\begin{tabular}{|c|c|c|c|c|c|}
\hline Familiarity condition & Validity condition & $\begin{array}{l}\text { Mean difference of } \\
\text { SOA (ms) }\end{array}$ & $\begin{array}{l}\text { Pooled standard } \\
\text { deviation (ms) }\end{array}$ & Cohen's d & $\begin{array}{c}\text { Confidence } \\
\text { interval }\end{array}$ \\
\hline Familiar & Invalid & 13.7 & 60.0 & 0.23 & $0.14,0.35$ \\
\hline Familiar & Valid & 32.1 & 69.1 & 0.46 & $0.37,0.57$ \\
\hline Unfamiliar & Invalid & 6.47 & 63.1 & 0.10 & $0.02,0.20$ \\
\hline Unfamiliar & Valid & 12.5 & 72.0 & 0.17 & $0.09,0.27$ \\
\hline
\end{tabular}

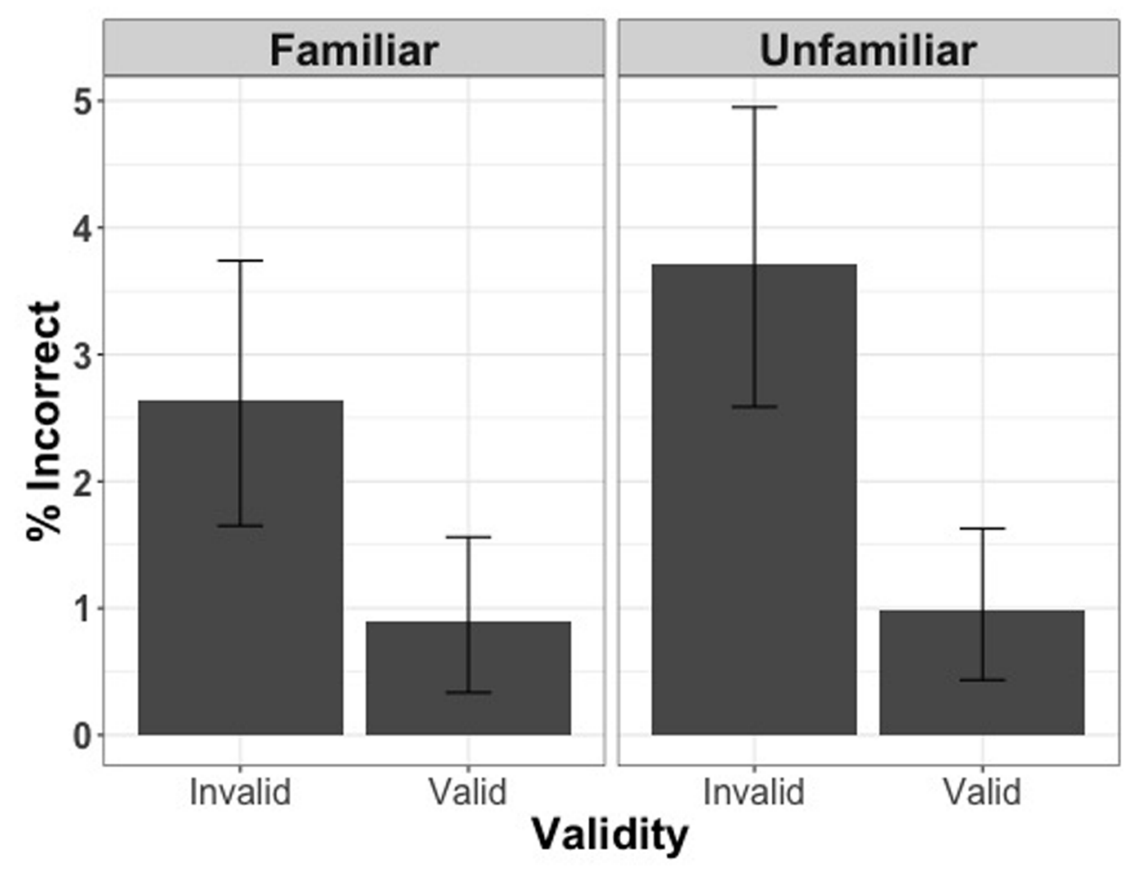

FIGURE 4 | Percentage of incorrect responses for valid and invalid trials when the face cue was familiar or unfamiliar in Experiment 2.

written informed consent to participate in the experiment, and were paid. The Dartmouth Committee for the Protection of Human Subjects approved the experiment (Protocol 21200).

\section{Experimental Paradigm}

The experimental paradigm was similar to Experiments 1 and 2, except that there was no target following the change in eye gaze. Participants performed three blocks of 50 trials each, resulting in a total of 150 trials. Over the course of the experiment, the picture of each individual identity was repeated 25 times.

Participants were instructed to press either the left arrow or the right arrow key to indicate the direction of the eye gaze change (either to the left or to the right) of the centrally presented face. As in the first two experiments, participants were instructed to be as fast as possible in their response.

\section{Data Analysis}

We rejected trials with reaction times less than $100 \mathrm{~ms}$.

We constructed a linear mixed model with log transformed manual response time as the dependent variable, the familiarity condition as the fixed effect and the participants as random effects. The values reported in the results were obtained from
Type 3 Analysis of Deviance on each model, performed with the function ANOVA from package car (Fox and Weisberg, 2010).

\section{Results}

The linear mixed model revealed a significant effect of familiarity condition on reaction time for reporting the direction of changes in eye gaze direction $\left[\chi^{2}(1)=39.75, p<0.001\right]$, with shorter reaction times for familiar faces $(M=425 \mathrm{~ms}, C I=[420 \mathrm{~ms}$, $430 \mathrm{~ms}])$ than for unfamiliar faces $(M=450 \mathrm{~ms}, \mathrm{CI}=[445 \mathrm{~ms}$, $454 \mathrm{~ms}]$ ) (Figure 6). Cohen's $d$ for this effect was 0.53 . There was no effect of familiarity on accuracy $\left[\chi^{2}(1)=0.76, p=0.38\right]$.

\section{Interim Discussion}

The results of this experiment, in line with the findings of Visconti di Oleggio Castello et al. (2014), show faster processing of eye gaze in personally familiar as compared to unfamiliar faces. These results further support the hypothesis that slower response to targets with personally familiar face cues as compared to unfamiliar face cues, is due to the holding of attention by the personally familiar faces rather than to slower processing of eye gaze shifts. 


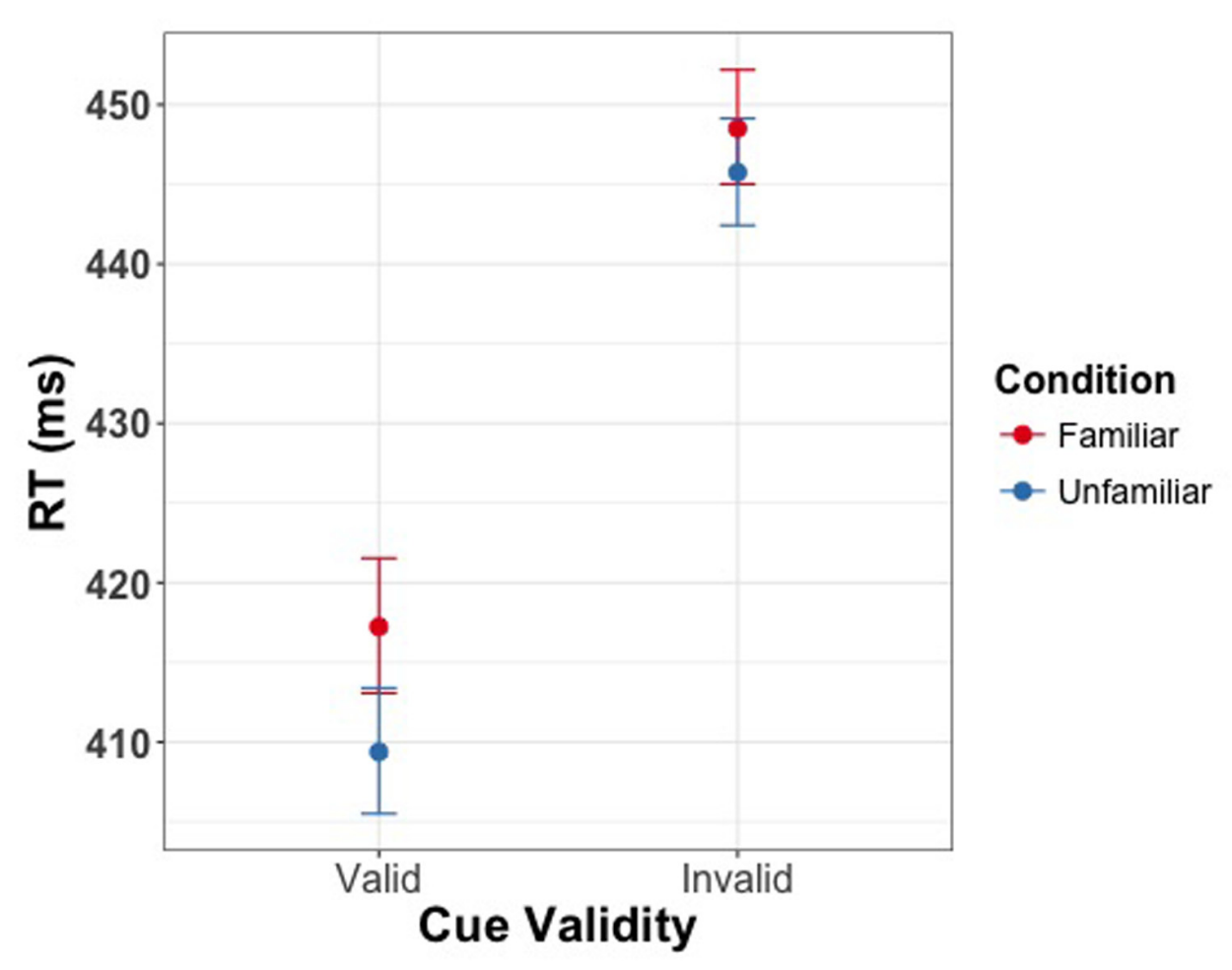

FIGURE 5 | Manual response time as a function of validity of the gaze cue in Experiment 2. Error bars represent bootstrapped $95 \%$ confidence intervals around the mean. Participants were slower on invalid trials, and their latency was affected by the familiarity of the cues.

\section{DISCUSSION}

We tested the interaction between reallocation of spatial attention mediated by shifts in eye gaze and personal familiarity with faces. We used an adapted version of the Posner paradigm to study attention shifts elicited by eye gaze movements in others. Overall, the results showed facilitation of responses to peripheral targets in trials with congruent cues as compared to trials with incongruent cues. These results replicate the effect of cue validity on saccadic response time found in prior research (Kuhn and Kingstone, 2009). Consistent with earlier studies, reaction times were faster and the cue validity effect was larger at the longer SOA of $200 \mathrm{~ms}$ (Friesen and Kingstone, 1998; Kuhn and Kingstone, 2009). Here, we report for the first time an interaction of SOA and personal familiarity wherein face familiarity slows the redirection of spatial attention mediated by eye gaze at short but not long SOAs. More specifically, in our first experiment we found that gaze shifts in personally familiar faces, as compared to in unfamiliar faces, elicited slower saccadic response times at a short SOA of $100 \mathrm{~ms}$ between the gaze shift and the onset of the peripheral target. Familiarity did not modulate the effect of cue validity. No modulation by familiarity was recorded at the longer SOA of $200 \mathrm{~ms}$, indicating that the slowing of attention shifts due to cue face familiarity is brief. The results of the second experiment, which required maintaining fixation on the cue face and manual responses, showed that the effect of familiarity was not specific to saccadic responses that required looking away from the face.
The size of the familiarity effect, however, was smaller than for saccadic responses. The additional time to prepare a manual response (over $150 \mathrm{~ms}$ ) may diminish the familiarity effect, which appears to be rapidly fading.

The third experiment investigated whether the results reported in Experiments 1 and 2 were due to differences in processing of eye gaze in familiar and unfamiliar faces, rather than to differences in the holding of attention by familiar and unfamiliar faces. Results from Experiment 3 showed that personal familiarity significantly facilitated detection of the direction of gaze shifts, as compared to unfamiliar faces, indicating that the familiarity effect on gaze-cued attention shifts to the periphery, which was in the opposite direction, is not due to slower detection of the gaze cue itself. Interestingly, the facilitation of eye gaze detection by familiarity appears to be stronger for more demanding tasks. In a visual search task, we found a larger effect of facilitation on RT, over $100 \mathrm{~ms}$ (Visconti di Oleggio Castello et al., 2014). By contrast, we found only a non-significant trend toward facilitation in a simple gaze change detection task (see Supplementary Material) unlike the task in Experiment 3 that required indicating the gaze change direction. Therefore, despite facilitated detection of eye gaze shifts in familiar faces, reallocation of attention away from the face is slowed by personal familiarity due to slowed disengagement of attention.

To summarize, our results indicate that familiarity delays gaze-cued attentional shifts at short latencies by briefly slowing deployment of attention away from familiar faces. Our results 
TABLE 4 | Main effect of familiarity in Experiment 2: Familiar RT - Unfamiliar RT.

\begin{tabular}{lcccc}
\hline $\begin{array}{l}\text { Validity } \\
\text { condition }\end{array}$ & $\begin{array}{c}\text { Mean } \\
\text { difference of } \\
\text { familiarity (ms) }\end{array}$ & $\begin{array}{c}\text { Pooled } \\
\text { standard } \\
\text { deviation (ms) }\end{array}$ & Cohen's d & $\begin{array}{c}\text { Confidence } \\
\text { interval }\end{array}$ \\
\hline Invalid & 5.04 & 50.2 & 0.10 & $-0.06,0.21$ \\
Valid & 6.85 & 60.4 & 0.11 & $0.02,0.23$
\end{tabular}

TABLE 5 | Main effect of cue validity in Experiment 2: Invalid RT - Valid RT.

\begin{tabular}{lcccc}
\hline $\begin{array}{l}\text { Familiarity } \\
\text { condition }\end{array}$ & $\begin{array}{c}\text { Mean } \\
\text { difference of } \\
\text { cue validity (ms) }\end{array}$ & $\begin{array}{c}\text { Pooled } \\
\text { standard } \\
\text { deviation }(\mathbf{m s})\end{array}$ & Cohen's d & $\begin{array}{c}\text { Confidence } \\
\text { interval }\end{array}$ \\
\hline Familiar & 32.0 & 54.3 & 0.59 & $0.50,0.69$ \\
Unfamiliar & 33.9 & 50.2 & 0.67 & $0.59,0.78$ \\
\hline
\end{tabular}

suggest that the familiar faces capture attention (Simons, 2000), and that this effect fades quickly. Slowed disengagement of attention from the familiar faces overrides any advantage of faster detection of gaze changes in familiar, as compared to unfamiliar faces. Holding of attention by personally familiar faces also has been shown by others with a different experimental paradigm, namely visual search (Devue et al., 2009).

The relationship of social salience and bias of spatial attention has been studied using personally familiar faces but with a different degree of familiarity as compared to the personally familiar faces chosen for our experiments. In previous studies, faces of colleagues or coworkers have been used as familiar stimuli. For example, Hungr and Hunt (2012) used one's own face and a familiar confederate -the face of the experimenter- as familiar faces, and Deaner et al. (2007) used faces of professors, postdoctoral researchers and graduate students from the same department of the participants. Hungr and Hunt (2012) reported faster reaction times in the gaze cueing paradigm for the confederate's faces, and longer for one's own face and for faces of strangers. Deaner et al. (2007) reported a complex interaction for gender and familiarity with faster reaction times in women when participants were cued by familiar faces. In our experiments, we aimed to study the effect of familiarity that is characterized by a personal, close relationship with repeated social interactions over time rather than simple prior visual exposure. Personally familiar individuals with whom we have a close relationship have a special status and are processed more efficiently as compared to other type of familiar faces, such as famous faces and visually familiar faces. Therefore, we chose as stimuli, the faces of friends with whom the participants had a good close relationship for at least a year.

Our results provide evidence for slowed disengagement of attention from personally familiar faces, a highly salient social stimulus. The effect of slowed disengagement of attention was only found for familiar faces at a short delay between the cue and the target. Interestingly, studies of blocking by salient cues in associative learning (Denton and Kruschke, 2006; Le Pelley et al., 2014) and attentional capture by cues of high valence (Hickey et al., 2010; Anderson et al., 2011) lend support to the idea that

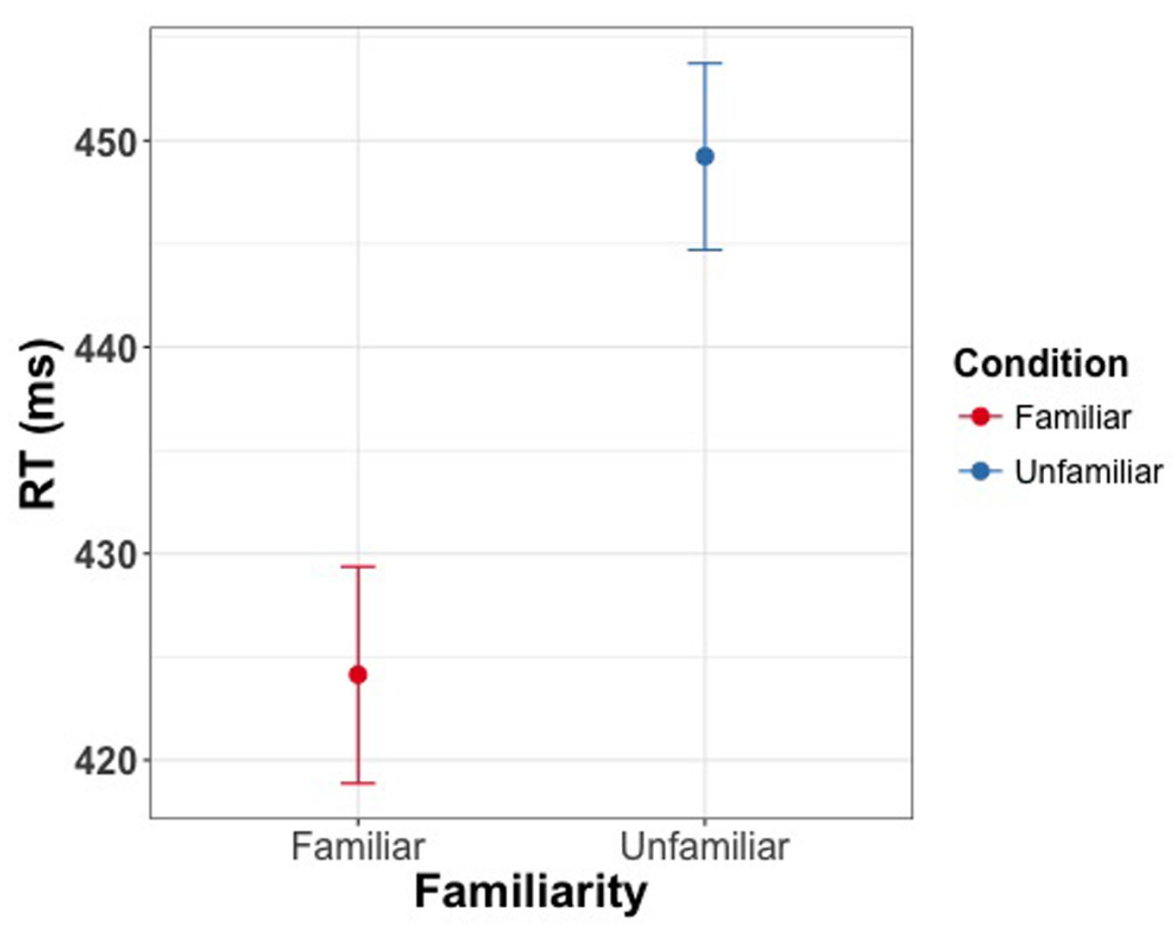

FIGURE 6 | Manual response times as a function of familiarity of the face cue in Experiment 4 . Participants were asked to indicate direction of eye gaze via button press. Participants were faster in detecting the direction of gaze for familiar faces. Error bars represent bootstrapped $95 \%$ confidence intervals around the mean. 
a centrally presented cue that is highly salient, as compared to cues of less salience, leads to slower redirection of attention rather than serving as a more informative cue. Our results are consistent with these studies that used cues other than faces. Repeated exposure to the faces of familiar individuals and the semantic and emotional information associated with these identities make them socially salient. In our study, we demonstrate that this highly salient social cue holds attention rather than facilitates redirection of attention.

\section{AUTHOR CONTRIBUTIONS}

MIG conceived the idea for the study. VC, MIG, and AS designed the experiment. VC collected and analyzed the data. MVdOC provided suggestions for data analysis. VC prepared the

\section{REFERENCES}

Anderson, B. A., Laurent, P. A., and Yantis, S. (2011). Value-driven attentional capture. Proc. Natl. Acad. Sci. U.S.A. 108, 10367-10371. doi: 10.1073/pnas. 1104047108

Bates, D., Mächler, M., Bolker, B., and Walker, S. (2014). Fitting linear mixed-effects models using lme4. arXiv 1406, 5823.

Bindemann, M., Burton, A. M., Hooge, I. T., Jenkins, R., and de Haan, E. H. (2005). Faces retain attention. Psychon. Bull. Rev. 12, 1048-1053. doi: 10.3758/ BF03206442

Bruce, V., Burton, M. A., and Dench, N. (1994). What's distinctive about a distinctive face? Q. J. Exp. Psychol. 47, 119-141. doi: 10.1080/ 14640749408401146

Burton, A. M., Wilson, S., Cowan, M., and Bruce, V. (1999). Face recognition in poor-quality video: evidence from security surveillance. Psychol. Sci. 10, 243-248. doi: 10.1111/1467-9280.00144

Carlin, J. D., and Calder, A. J. (2013). The neural basis of eye gaze processing. Curr. Opin. Neurobiol. 23, 450-455. doi: 10.1016/j.conb.2012.11.014

Carlin, J. D., Rowe, J. B., Kriegeskorte, N., Thompson, R., and Calder, A. J. (2012). Direction-sensitive codes for observed head turns in human superior temporal sulcus. Cereb. Cortex 22, 735-744. doi: 10.1093/cercor/bhr061

Champely, S. (2009). pwr: Basic Functions for Power Analysis. R Package Version 1.1. 1. Vienna: The R Foundation.

Deaner, R. O., Shepherd, S. V., and Platt, M. L. (2007). Familiarity accentuates gaze cuing in women but not men. Biol. Lett. 3, 65-68. doi: 10.1098/rsbl.2006.0564

Denton, S. E., and Kruschke, J. K. (2006). Attention and salience in associative blocking. Learn. Behav. 34, 285-304. doi: 10.3758/BF03192884

Devue, C., Van der Stigchel, S., Brédart, S., and Theeuwes, J. (2009). You do not find your own face faster; you just look at it longer. Cognition 111, 114-122. doi: 10.1016/j.cognition.2009.01.003

Driver, J., Davis, G., and Ricciardelli, P. (1999). Gaze perception triggers reflexive visuospatial orienting. Vis. Cogn. 6, 509-540. doi: 10.1080/135062899394920

Engell, A. D., and Haxby, J. V. (2007). Facial expression and gaze-direction in human superior temporal sulcus. Neuropsychologia 45, 3234-3241. doi: 10.1016/j.neuropsychologia.2007.06.022

Fox, J., and Weisberg, S. (2010). An R Companion to Applied Regression. Thousand Oaks, CA: Sage.

Friesen, C. K., and Kingstone, A. (1998). The eyes have it! Reflexive orienting is triggered by nonpredictive gaze. Psychon. Bull. Rev. 5, 490-495. doi: 10.3758/ BF03208827

Frischen, A., Bayliss, A. P., and Tipper, S. P. (2007). Gaze cueing of attention: visual attention, social cognition, and individual differences. Psychol. Bull. 133, 694. doi: 10.1037/0033-2909.133.4.694

Gobbini, M. I., Gors, J. D., Halchenko, Y. O., Hughes, H. C., and Cipolli, C. (2013a). Processing of invisible social cues. Conscious. Cogn. 22, 765-770. doi: 10.1016/j.concog.2013.05.002 manuscript, MIG, MVdOC, and AS provided critical inputs to the final version of the manuscript.

\section{ACKNOWLEDGMENTS}

We thank Morgan Taylor for data collection of Experiment 3. We thank Jim Haxby and Carlo Cipolli for helpful comments and discussion.

\section{SUPPLEMENTARY MATERIAL}

The Supplementary Material for this article can be found online at: http://journal.frontiersin.org/article/10.3389/fpsyg. 2017.00738/full\#supplementary-material

Gobbini, M. I., Gors, J. D., Halchenko, Y. O., Rogers, C., Guntupalli, J. S., Hughes, H., et al. (2013b). Prioritized detection of personally familiar faces. PLoS ONE 8:e66620. doi: 10.1371/journal.pone.0066620

Gobbini, M. I., and Haxby, J. V. (2007). Neural systems for recognition of familiar faces. Neuropsychologia 45, 32-41. doi: 10.1016/j.neuropsychologia.2006.04.015

Hickey, C., Chelazzi, L., and Theeuwes, J. (2010). Reward changes salience in human vision via the anterior cingulate. J. Neurosci. 30, 11096-11103. doi: 10.1523/JNEUROSCI.1026-10.2010

Hickey, C., and Peelen, M. V. (2015). Neural mechanisms of incentive salience in naturalistic human vision. Neuron 85, 512-518. doi: 10.1016/j.neuron.2014. 12.049

Hoffman, E. A., and Haxby, J. V. (2000). Distinct representations of eye gaze and identity in the distributed human neural system for face perception. Nat. Neurosci. 3, 80-84. doi: 10.1038/71152

Hungr, C. J., and Hunt, A. R. (2012). Physical self-similarity enhances the gazecueing effect. Q. J. Exp. Psychol. 65, 1250-1259. doi: 10.1080/17470218.2012. 690769

Itti, L., and Koch, C. (2001). Computational modelling of visual attention. Nat. Rev. Neurosci. 2, 194-203. doi: 10.1038/35058500

Jenkins, R., White, D., Van Montfort, X., and Burton, A. M. (2011). Variability in photos of the same face. Cognition 121, 313-323. doi: 10.1016/j.cognition.2011. 08.001

Kawai, N. (2011). Attentional shift by eye gaze requires joint attention: eye gaze cues are unique to shift attention. Jpn. Psychol. Res. 53, 292-301. doi: 10.1111/j. 1468-5884.2011.00470.x

Khorsand, P., Moore, T., and Soltani, A. (2015). Combined contributions of feedforward and feedback inputs to bottom-up attention. Front. Psychol. 6:155. doi: 10.3389/fpsyg.2015.00155

Kleiner, M., Brainard, D., Pelli, D., Ingling, A., Murray, R., and Broussard, C. (2007). What's new in Psychtoolbox-3. Perception 36, 1-16.

Kuhn, G., and Kingstone, A. (2009). Look away! Eyes and arrows engage oculomotor responses automatically. Atten. Percept. Psychophys. 71, 314-327. doi: 10.3758/APP.71.2.314

Langton, S. R., and Bruce, V. (1999). Reflexive visual orienting in response to the social attention of others. Vis. Cogn. 6, 541-567. doi: 10.1080/135062899394939

Le Pelley, M. E., Beesley, T., and Griffiths, O. (2014). Relative salience versus relative validity: cue salience influences blocking in human associative learning. J. Exp. Psychol. 40, 116. doi: 10.1037/xan0000006

Markowitz, D. A., Shewcraft, R. A., Wong, Y. T., and Pesaran, B. (2011). Competition for visual selection in the oculomotor system. J. Neurosci. 31, 9298-9306. doi: 10.1523/JNEUROSCI.0908-11.2011

Munneke, J., Hoppenbrouwers, S. S., and Theeuwes, J. (2015). Reward can modulate attentional capture, independent of top-down set. Atten. Percept. Psychophys. 77, 2540-2548. doi: 10.3758/s13414-015-0958-6

Pageler, N. M., Menon, V., Merin, N. M., Eliez, S., Brown, W. E., and Reiss, A. L. (2003). Effect of head orientation on gaze processing in fusiform gyrus 
and superior temporal sulcus. Neuroimage 20, 318-329. doi: 10.1016/S10538119(03)00229-5

Pelphrey, K. A., Morris, J. P., and McCarthy, G. (2005). Neural basis of eye gaze processing deficits in autism. Brain 128, 1038-1048. doi: 10.1093/brain/awh404

Pelphrey, K. A., Singerman, J. D., Allison, T., and McCarthy, G. (2003). Brain activation evoked by perception of gaze shifts: the influence of context. Neuropsychologia 41, 156-170. doi: 10.1016/S0028-3932(02)00146-X

Pérez-Duenas, C., Acosta, A., and Lupiánez, J. (2014). Reduced habituation to angry faces: increased attentional capture as to override inhibition of return. Psychol. Res. 78, 196-208. doi: 10.1007/s00426-013-0493-9

Porciello, G., Holmes, B. S., Liuzza, M. T., Crostella, F., Aglioti, S. M., and Bufalari, I. (2014). Interpersonal multisensory stimulation reduces the overwhelming distracting power of self-gaze: psychophysical evidence for “engazement”. Sci. Rep. 4:6669. doi: 10.1038/srep06669

Posner, M. I. (1980). Orienting of attention. Q. J. Exp. Psychol. 32, 3-25. doi: 10.1080/00335558008248231

Pourtois, G., Schettino, A., and Vuilleumier, P. (2013). Brain mechanisms for emotional influences on perception and attention: what is magic and what is not. Biol. Psychol. 92, 492-512. doi: 10.1016/j.biopsycho.2012.02.007

R Core Team (2013). R: A Language and Environment for Statistical Computing. Vienna: R Core Team.

Ramon, M. (2015). Perception of global facial geometry is modulated through experience. PeerJ 3, e850. doi: 10.7717/peerj.850

Ramon, M., Caharel, S., and Rossion, B. (2011). The speed of recognition of personally familiar faces. Perception 40, 437-449. doi: 10.1068/p6794

Ramon, M., and Van Belle, G. (2016). Real-life experience with personally familiar faces enhances discrimination based on global information. PeerJ 4, e1465. doi: $10.7717 /$ peerj.1465

Ricciardelli, P., Bricolo, E., Aglioti, S. M., and Chelazzi, L. (2002). My eyes want to look where your eyes are looking: exploring the tendency to imitate another individual's gaze. Neuroreport 13, 2259-2264. doi: 10.1097/00001756200212030-00018

Senju, A., and Csibra, G. (2008). Gaze following in human infants depends on communicative signals. Curr. Biol. 18, 668-671. doi: 10.1016/j.cub.2008. 03.059

Senju, A., and Johnson, M. H. (2009). The eye contact effect: mechanisms and development. Trends Cogn. Sci. 13, 127-134. doi: 10.1016/j.tics.2008.11.009

Simons, D. J. (2000). Attentional capture and inattentional blindness. Trends Cogn. Sci. 4, 147-155. doi: 10.1016/S1364-6613(00)01455-8

Soltani, A., and Koch, C. (2010). Visual saliency computations: mechanisms, constraints, and the effect of feedback. J. Neurosci. 30, 12831-12843. doi: 10.1523/JNEUROSCI.1517-10.2010
Stein, T., Senju, A., Peelen, M. V., and Sterzer, P. (2011). Eye contact facilitates awareness of faces during interocular suppression. Cognition 119, 307-311. doi: 10.1016/j.cognition.2011.01.008

Teufel, C., Alexis, D. M., Clayton, N. S., and Davis, G. (2010). Mental-state attribution drives rapid, reflexive gaze following. Atten. Percept. Psychophys. 72, 695-705. doi: 10.3758/APP.72.3.695

Theeuwes, J., and Belopolsky, A. V. (2012). Reward grabs the eye: oculomotor capture by rewarding stimuli. Vis. Res. 74, 80-85. doi: 10.1016/j.visres.2012. 07.024

Visconti di Oleggio Castello, M., and Gobbini, M. I. (2015). Familiar face detection in $180 \mathrm{~ms}$. PLoS ONE 10:e0136548. doi: 10.1371/journal.pone.0136548

Visconti di Oleggio Castello, M., Guntupalli, J. S., Yang, H., and Gobbini, M. I. (2014). Facilitated detection of social cues conveyed by familiar faces. Front. Hum. Neurosci. 8:678. doi: 10.3389/fnhum.2014.00678

Visconti di Oleggio Castello, M., Wheeler, K. G., Cipolli, C., and Gobbini, M. I. (2016). Familiarity facilitates feature-based face processing. bioRxiv 058537. doi: 10.1101/058537

Wiese, E., Wykowska, A., and Muller, H. J. (2014). What we observe is biased by what other people tell us: beliefs about the reliability of gaze behavior modulate attentional orienting to gaze cues. PLoS ONE 9:e94529. doi: 10.1371/journal. pone.0094529

Wiese, E., Wykowska, A., Zwickel, J., and Muller, H. J. (2012). I see what you mean: how attentional selection is shaped by ascribing intentions to others. PLoS ONE 7:e45391. doi: 10.1371/journal.pone.0045391

Willenbockel, V., Sadr, J., Fiset, D., Horne, G. O., Gosselin, F., and Tanaka, J. W. (2010). Controlling low-level image properties: the SHINE toolbox. Behav. Res. Methods 42, 671-684. doi: 10.3758/BRM.42.3.671

Wykowska, A., Wiese, E., Prosser, A., and Muller, H. J. (2014). Beliefs about the minds of others influence how we process sensory information. PLoS ONE 9:e94339. doi: 10.1371/journal.pone.0094339

Conflict of Interest Statement: The authors declare that the research was conducted in the absence of any commercial or financial relationships that could be construed as a potential conflict of interest.

Copyright (c) 2017 Chauhan, Visconti di Oleggio Castello, Soltani and Gobbini. This is an open-access article distributed under the terms of the Creative Commons Attribution License (CC BY). The use, distribution or reproduction in other forums is permitted, provided the original author(s) or licensor are credited and that the original publication in this journal is cited, in accordance with accepted academic practice. No use, distribution or reproduction is permitted which does not comply with these terms. 